\title{
Juegos lúdicos para mejorar la motricidad gruesa en niños de 4 a 5 años
}

\section{Playful Games to Improve Gross Motor Skills in Children from 4 to 5 years old}

1 Danny Paul Landa Pico

(iD) https://orcid.org/0000-0001-6380- 5808

Universidad Técnica de Ambato, Facultad de Ciencias Humanas y de la Educación,

Carrera de Psicopedagogía), Ambato, Ecuador,

dlanda3175@uta.edu.ec

2 Gerson Stalin Chiliquinga Sifas

https://orcid.org/0000-0003-4240-9835

Universidad Técnica de Ambato, Facultad de Ciencias Humanas y de la Educación,

Carrera de Psicopedagogía), Ambato, Ecuador,

gchiliquinga2744@uta.edu.ec

3 Gissela Alexandra Arroba López

iD https://orcid.org/0000- 0002-7846-6535

Universidad Técnica de Ambato, Facultad de Ciencias Humanas y de la Educación,

Carrera de Educación Inicial) Ambato, Ecuador

ga.arroba@uta.edu.ec

4 Tamara Yajaira Ballesteros Casco iD https://orcid.org/ 0000- 0002-5962-7962

Universidad Técnica de Ambato, Facultad de Ciencias Humanas y de la Educación,

Carrera de Educación Inicial) Ambato, Ecuador

ty.ballesteros@uta.edu.ec

Artículo de Investigación Científica y Tecnológica

Enviado: 24/12/2021

Revisado: 29/12/2021

Aceptado: $12 / 01 / 2022$

Publicado:08/03/2023

DOI: https://doi.org/10.33262/concienciadigital.v6i1.4.2010

Landa Pico, D. P., Chiliquinga Sifas, G. S., Arroba López, G. A., \& Ballesteros Casco, T.

Cítese: $\quad$ Y. (2023). Juegos lúdicos para mejorar la motricidad gruesa en niños de 4 a 5 años. ConcienciaDigital, $6(1.4)$,

489-505. https://doi.org/10.33262/concienciadigital.v6i1.4.2010

CONCIENCIA DIGITAL, es una Revista Multidisciplinar, Trimestral, que se publicará en soporte electrónico tiene como misión contribuir a la formación de profesionales competentes con visión humanística y crítica que sean capaces de exponer sus resultados investigativos y científicos en la misma medida que se promueva mediante su intervención cambios positivos en la sociedad. https://concienciadigital.org .

La revista es editada por la Editorial Ciencia Digital (Editorial de prestigio registrada en la Cámara Ecuatoriana de Libro con No de Afiliación 663) www.celibro.org.ec 


\section{Palabras}

claves:

lúdica, desarrollo motor grueso, infantes, relación directa, autonomía.

\section{Keywords:}

playful, gross motor development, infants, direct relationship, autonomy.

\section{Resumen}

Introducción. El desarrollo humano se caracteriza por cumplir diferentes procesos específicos que se van dando a través del crecimiento, dentro de estas las más básicas como caminar, trotar, correr, levantarse son distintivas por estar dentro del desarrollo motor grueso, este como un eje fundamental para que el niño tenga autonomía de movimiento, así mismo se habla que la base que motiva a los infantes es el juego. En la presente investigación se trata de correlacionar como el uso actividades lúdicas, las cuales toman la forma de juegos específicos pueden ayudar a los niños a mejorar el desarrollo motor grueso por estar relacionado a la capacidad que el niño tiene para moverse, así mismo como se pueden usar estas técnicas dentro del proceso educativo escolar. Objetivo. Analizar la relación existente entre los juegos lúdicos y el desarrollo motor grueso de niños entre 4 y 5 años. Metodología. La presente investigación tiene el carácter descriptivo, con un enfoque cualitativo, el cual se basa en una revisión bibliográfica que permite comprender la influencia de las actividades lúdicas con relación al desarrollo de la motricidad gruesa. Resultados. Se ha logrado evidenciar que el uso de las actividades lúdicas tiene una relación directa con el desarrollo motor grueso de los niños, viéndose así que es un factor fundamental para aplicarse para mejorar la autonomía, desarrollo de movimiento y coordinación, en niños de la edad propuesta.

\section{Abstract}

Introduction. Human development is characterized by fulfilling different specific processes that are given through growth, within these the most basic ones like walking, jogging, running, rising are distinctive because they are within the thick motor development, this is a fundamental axis for the child to have autonomy of movement, and it is also said that the foundation that motivates infants is play. This research is about correlating how the use of recreational activities, which take the form of specific games, can help children improve thick motor development by being related to the ability of the child to move. as well as how these techniques can be used within the school educational process. Objective. To analyze the relationship between play and the gross motor development of children between 4 and 5 years of age. Methodology. The present research has a descriptive character, 
with a qualitative approach, which is based on a bibliographic review that allows to understand the influence of recreational activities about the development of gross motor. Results. It has been demonstrated that the use of recreational activities has a direct relationship with the gross motor development of children, thus being seen as a fundamental factor to be applied to improve autonomy, development of movement and coordination in children of the proposed age.

\section{Introducción}

\section{La Lúdica}

El sinónimo más utilizado en cuanto a la lúdica es el Juego en este caso. El juego es un tipo de actividad que no tiende a ser una necesidad biológica humana, ya que esta no tiene un objetivo de aplicación a la vida diaria, sin embargo, es necesario entender cuál es el objetivo del uso de la lúdica dentro del desarrollo infantil.

Al juego se lo menciona: "Como una actividad libre, que mantiene un aspecto relativamente asociado a la realidad y que es capaz de comprometer al participante dentro del mismo".

Para Sanjinés (2018) "El juego proviene del latín locus cuyo término se define como la acción de jugar, bromear.” (p. 22). Con base en esta definición se piensa que le juego se atribuye solo a divertirse. Sin embargo, en Balseca y Grace (2016) describe: "El juego para los niños es una actividad que produce placer, el cual permite realizar cualquier actividad sin un deterioro cognitivo".

En este mismo texto, se permite explicar que la actividad lúdica que niños realizan crea conocimiento del contexto y permiten reconocer sus capacidades, es por esto por lo que los estudios relacionados al juego ayudan identificar el grado de cognición y permite visualizar que las habilidades estimuladas en este caso son: simbólicas, motrices, sensoriales y de razonamiento.

Se ha definido que el juego es una actividad social especifica realizada por los seres humanos, además que esta puede estar presente durante toda la vida sin importar edad. Sin embargo, esta es más observable durante la primera infancia, en donde los niños tienen mayor motivación de lo que están haciendo ya que lo hacen mientras usan el juego como medio de interacción con el entorno. Tomado lo dicho anteriormente para Macias (2017) la interacción del maestro y el estudiante dentro del proceso enseñanza y aprendizaje tiene que constar la reciprocidad y que el componente contextual esté vinculado socialmente.

Para Balseca y Grace (2016) sobre el juego y la motricidad gruesa se menciona que 
Vygotsky infiere que juego puede ser usado como instrumento o también como un recurso a nivel sociocultural, definiendo que el juego puede impulsar el desarrollo cognitivo del niño, esto facilita el desarrollo de diferentes capacidades como: la atención y memoria, en cuanto al juego se crea aprendizaje, y le permite al niño expresarse y regular las emociones.

Existe otra forma de desarrollo fuera del socio- afectivo cuando se habla de lúdica en este caso, se habla del desarrollo psicomotriz mediante el uso de movimientos como: correr, saltar, caminar, entre otras actividades estos movimientos ayudan a los niños a coordinarse, equilibrarse, orientarse en relación con sus cuerpos en espacio y tiempo este tipo de acciones se atribuyen al termino motricidad gruesa (Preciado, 2019).

\section{La lúdica y el desarrollo}

Para Calero et. al (2020) los juegos se los consideran como actividades muy primordiales para el desarrollo cognitivo y social para los seres humanos desde edades muy tempranas e incluso para edades avanzadas. Como adición La lúdica o el juego no solo es importante para la capacidad intelectual, sino que además ayuda a potenciar los valores humanos como pueden ser: la motricidad, la inteligencia, la creatividad y la sociabilidad.

\section{La lúdica como un medio de aprendizaje}

Dentro del uso de la lúdica existen diversos objetivos entre estos están: generar un estímulo de acción, reflexión y una forma de expresión propia. Se puede así mismo considerar a la lúdica como un medio que permite explorar, crear y descubrir la razón del porque pasa una u otra cosa en su entorno, es bastante considerada la idea de aprender jugando ya que se nos permite contemplar cual es el fundamento psicológico del uso del juego como forma de estudio, la razón principal es que el juego les da una libertad constante de recibir la información sin una imposición, en este caso el juego también propone el dar placer o satisfacción durante el proceso de aplicación contemplando así el principio de asociación por estímulos positivos.

\section{Beneficios del uso de la lúdica}

Para Ramírez (2019), principalmente el uso de la lúdica se direcciona a un modelo de enseñanza aprendizaje, pero este también puede ser usado como un método de control de la conducta, motivación y orientación, se ha propuesto una serie de rasgos que determinan que tan bien se aplica la lúdica a nivel educativo, los cuales se presentan a continuación:

- Potenciamiento de la creatividad.

- Permitir el desarrollo global.

- Que sea gratificante para los participantes. 
- Evitar actividades de eliminación.

- Controlar el uso excesivo de la competitividad.

Según Lupuche (2017) indica que las actividades lúdicas son aquellas que nos permiten entender y conocer la importancia del desarrollo del niño o la niña en canto se refiere a su cuerpo corporal y con relación a la edad. También el juego se atribuye un papel primordial en el desarrollo del infante, donde se favorece la independencia, el desarrollo de la motricidad gruesa, tanto en el equilibrio y coordinación del cuerpo humano.

\section{Motricidad}

Para Ramírez (2019) la motricidad implica todo movimiento que puede realizar el ser vivo desde pequeño, esto se lo puede evidenciar como habilidades o destrezas en distintos movimientos, llevando a cabo, de lo más simple hasta lo más complejo, la Motricidad gruesa hace relación a una diversidad de movimientos más precisos en lo que engloba a coordinación del cuerpo con el razonamiento o la razón. Dicho de otro modo, el cuerpo se va a mover a voluntad propia.

Dentro del crecimiento del niño se ha visto esencial un desarrollo individual de la motricidad corporal, dando lugar a la capacidad de controlar movimientos específicos, generar habilidades de coordinación y una correcta funcionalidad de la masa corporal, esto gracias a estructuras talámicas y subtalámicas que son el centro de control motor (Rigal, 2016).

\section{Motricidad gruesa}

Se define como todo proceso donde interviene el cuerpo este tipo de acciones pueden se: mantener el equilibrio, coordinación y lateralidad, se habla así mismo que es la sincronía existente al realizar movimientos de grandes grupos musculares, óseos y nerviosos, este tipo de movimiento sincrónico esta descrito dentro de actividades que los niños realizan comúnmente (Gutiérrez, 2013).

Con respecto a la motricidad gruesa Chocce y Conde (2018) describen que la motricidad gruesa o conocida también como motricidad global se define al control de los movimientos musculares de nuestro cuerpo en general, estos movimientos llevan al niño a desplazarse de manera autónoma como, por ejemplo: control de la cabeza, girar sobre su propio eje, gatear, sentarse, caminar, correr, mantenerse se pie o lanzar objetos. Todo esto implica motricidad gruesa y tener la habilidad para realizar dichos movimientos. 


\section{Tabla 1}

Dimensiones de la Motricidad Gruesa

\begin{tabular}{|c|c|c|}
\hline Variable & Dimensión & Indicador \\
\hline \multirow{3}{*}{ Motricidad gruesa } & $\begin{array}{c}\text { Coordinación dinámica } \\
\text { general }\end{array}$ & $\begin{array}{l}\text { Este se caracteriza por utilizar } \\
\text { simultáneamente distintas partes } \\
\text { del cuerpo como: una carrera y } \\
\text { una marcha. Todo esto } \\
\text { conllevara del } \\
\text { equilibrio. }\end{array}$ \\
\hline & El tono muscular & $\begin{array}{l}\text { El musculo en estado de reposo } \\
\text { entra en contracción. Estas } \\
\text { acciones están comandadas por } \\
\text { el cerebelo }\end{array}$ \\
\hline & El control postural & 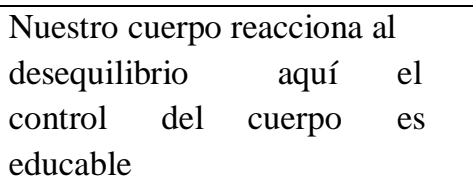 \\
\hline
\end{tabular}

Nota. Dimensiones de la motricidad gruesa

Fuente: Rigal (2016)

\section{Desarrollo motor grueso en los niños}

El desarrollo de la motricidad gruesa en el preescolar es de vital importancia y a lo largo del tiempo se involucrado a la educación física, buscando alcanzar la formación de un módulo más complejo denominada "educación integral", la misma que en la actualidad es un área poco profundizada en cuanto en cuanto a fines, métodos y orientación, esta es la educación motriz. La madurez motriz, fundamenta todos los aspectos del desarrollo integral del niño, puesto que fomenta globalmente todos los planos vitales que constituyen la mente y el cuerpo en general. De esta forma se emplea el concepto exploratorio de diferentes actores.

Al respecto Camargo y Maciel (2016) hacen ver su psicología genética como la necesidad de conexión entre lo orgánico, lo psíquico y el entorno, estableciendo un mundo "de relaciones simbióticas" determinándose entre la importancia del aspecto emocional en la evolución del individuo. La introducción de este aspecto es el lazo que une lo orgánico con lo psíquico y esta comunicación influirá en el tono postural del individuo, modificando indudablemente sus actitudes.

Para Vera (2019) el profesional parvulario tiene un papel importante dentro de la formación de niños en su etapa preescolar, mismos que deben elegir cual es la mejor estrategia que permita motivar he interactuar con sus pares y adultos. Dichas estrategias 
desarrollaran el aspecto corporal con movimientos musculares de: cabeza, abdomen, piernas, correr, caminar, mantener un equilibrio de su cuerpo en si entre otros. Los mencionados anterior mente necesitan de estimulación desde temprana edad y así lograran la madurez en sus movimientos gruesos y como no hacerlo atreves de la lúdica.

\section{El juego como estrategia en el desarrollo infantil}

Estela (2018) señala que el juego como es una de las actividades más antiguas del ser humano y ha contribuido en su evolución y desarrollo, a través del juego, los niños aprender a desarrollar y a fortalecer sus destrezas físicas y sociales. Durante el transcurso del juego el niño o la niña explora los diferentes roles familiares. El juego además orilla a que los niños aprendan a interactuar de manera social con los demás, dentro de las distintas actividades a realizar pueden ser tareas en equipo y surge la competitividad entre ellos.

El juego se considera como un medio de la Educación Física, por cuanto Díaz et al. (2014) afirma que a través del juego los estudiantes desde sus etapas iniciales de vida van formando las bases para un desarrollo armónico e integral. Agregando a lo mencionado anteriormente el juego permite desarrollar distintas habilidades y capacidades físicas que serán utilizadas en diferentes situaciones, para la solución de problemas que se presenten en el diario vivir.

Según Dente et al. (2018) el juego se lo comprende como un derecho humano de la infancia. Dicho de otra manera, por un lado, tenemos el Estado quien garantizará las políticas educativas donde se evidenciará proyectos y metas que contengan al juego por otra parte y siendo muy importante dentro de la comunidad educativa y dirigiéndonos específicamente a los docentes de Educación Física ellos podrán incluirlo en sus prácticas.

\section{Metodología}

La presente investigación tiene el carácter descriptivo, con un enfoque cualitativo, el cual se basa en una revisión bibliográfica que permite comprender la influencia de las actividades lúdicas con relación al desarrollo de la motricidad gruesa en los niños entre 4 a 5 años, durante su etapa escolar. La presente investigación realiza un contraste hacia la hipótesis planteada con el fin de medir cual es la incidencia de la primera variable (actividades lúdicas), con relación a la segunda (desarrollo de la psicomotricidad gruesa).

Se ha dispuesto que la presente investigación tiene un diseño no experimental, ya que no se usó un laboratorio, ni manipulación de variables en un ambiente controlado, en este caso se usa la observación y recolección de información como un medio de comprobación. La información recolectada se dio a nivel de campo en un solo momento de aplicación. 


\section{Resultados}

En este estudio se han tomado en cuenta como población a maestros y padres de familia de instituciones educativas de Ambato. La técnica empleada para la recolección fue una encuesta con 26 preguntas, subdivididas en dos áreas de medida, a lo cual se utiliza una escala de Likert diseñada en base a 5 opciones de respuesta donde se selecciona una sola opción.

\section{Tabla 2}

Escala de medición de las opciones de respuesta

\begin{tabular}{ccccc}
\hline \multicolumn{5}{c}{ Escala de medición. } \\
\hline 1 & 2 & 3 & 4 & 5 \\
Siempre & Generalmente & Ocasionalmente & Nunca & No observado
\end{tabular}

Fuente: Estudio de contexto

Se ha propuesto usar una escala de medición Likert con el fin de valorar las respuestas obtenidas en la presente investigación, notándose así que la obtención y calificación sea más específica.

\section{Tabla 3}

Batería instrumental para la medición de la motricidad de los niños de 4 a 5 años

\begin{tabular}{|c|c|c|c|c|c|c|c|c|c|}
\hline Descripción & Siempre & Gen & Imente & Oca & lmente & $\mathrm{Nu}$ & & No & ervado \\
\hline $\begin{array}{l}\text { 1. Camina con } \\
\text { movimientos } \\
\text { coordinados de los } \\
\text { brazos. }\end{array}$ & $54 \quad 68 \%$ & 20 & $20 \%$ & 5 & $6 \%$ & 1 & $1 \%$ & 0 & $0 \%$ \\
\hline $\begin{array}{l}\text { 2. Camina con } \\
\text { movimientos } \\
\text { coordinados de brazos }\end{array}$ & $4658 \%$ & 24 & $24 \%$ & 9 & $11 \%$ & 1 & $1 \%$ & 0 & $0 \%$ \\
\hline $\begin{array}{l}\text { 3. Camina por una } \\
\text { línea } \\
\text { dibujada en el piso. }\end{array}$ & $36 \quad 45 \%$ & 25 & $25 \%$ & 16 & $20 \%$ & 0 & $0 \%$ & 3 & $4 \%$ \\
\hline 4.Camina hacia atrás & $2734 \%$ & 15 & $15 \%$ & 29 & $36 \%$ & 6 & $8 \%$ & 3 & $4 \%$ \\
\hline $\begin{array}{l}\text { 5.Lanza la pelota de } \\
\text { arriba } \\
\text { asía abajo }\end{array}$ & $5063 \%$ & 25 & $25 \%$ & 2 & $3 \%$ & 2 & $3 \%$ & 1 & $1 \%$ \\
\hline $\begin{array}{l}\text { 6.Hace rodar la pelota } \\
\text { con } \\
\text { dos manos. }\end{array}$ & $48 \quad 60 \%$ & 19 & $19 \%$ & 13 & $16 \%$ & 0 & $0 \%$ & 0 & $0 \%$ \\
\hline $\begin{array}{l}\text { 7. Captura con ambas } \\
\text { manos y con ayuda del }\end{array}$ & $4455 \%$ & 18 & $18 \%$ & 18 & $23 \%$ & 0 & $0 \%$ & 0 & $0 \%$ \\
\hline
\end{tabular}




\section{Tabla 3}

Batería instrumental para la medición de la motricidad de los niños de 4 a 5 años

\begin{tabular}{|c|c|c|c|c|c|c|c|c|c|}
\hline \multirow{2}{*}{$\begin{array}{l}\text { Descripción } \\
\text { 8. Sube y desciende } \\
\text { una } \\
\text { silla de pequeña altura. }\end{array}$} & \multirow{2}{*}{$\begin{array}{l}\text { Siempre } \\
5873 \%\end{array}$} & \multicolumn{2}{|c|}{ Generalmente } & \multicolumn{2}{|c|}{ Ocasionalmente } & \multirow{2}{*}{$\begin{array}{c}\text { Nunca } \\
1\end{array}$} & \multicolumn{3}{|c|}{ No Observado } \\
\hline & & 17 & $17 \%$ & 4 & $5 \%$ & & $1 \%$ & 0 & $0 \%$ \\
\hline $\begin{array}{l}\text { 9. Se desplaza } \\
\text { arrastrando su cuerpo } \\
\text { por el piso, lleva } \\
\text { un brazo al frente }\end{array}$ & $3139 \%$ & 20 & $20 \%$ & 7 & $9 \%$ & 10 & $13 \%$ & 12 & $15 \%$ \\
\hline $\begin{array}{l}\text { 10. Se desplaza } \\
\text { separando } \\
\text { completamente el } \\
\text { cuerpo del piso } \\
\text { (gatea). }\end{array}$ & $3746 \%$ & 13 & $13 \%$ & 15 & $19 \%$ & 11 & $14 \%$ & 4 & $5 \%$ \\
\hline $\begin{array}{l}\text { 11. Se desplaza por el } \\
\text { piso } \\
\text { en cuatro puntos de } \\
\text { apoyo (pies y manos) }\end{array}$ & $3949 \%$ & 24 & $24 \%$ & 8 & $10 \%$ & 5 & $6 \%$ & 4 & $5 \%$ \\
\hline $\begin{array}{l}\text { 12. Realizar saltillos } \\
\text { laterales hacia un lado }\end{array}$ & $4253 \%$ & 21 & $21 \%$ & 14 & $18 \%$ & 2 & $3 \%$ & 1 & $1 \%$ \\
\hline
\end{tabular}

Fuente: Estudio de contexto

Los datos obtenidos por medio de la aplicación de la "Batería Instrumental Para la Medición de la Motricidad de los Niños de 4 a 5 Años" demuestran que los procesos básicos motrices que los niños de 4 y 5 años de edad mantiene un desarrollo normal, véase así que las respuestas en un alto porcentaje están descritas positivamente ya que se observa que más del $50 \%$ de los padres y docentes encuestados han observado claramente cuáles son las capacidades físicas de los niños dentro de este rango de edad.

\section{Tabla 4}

Cuestionario lúdica y desarrollo

\begin{tabular}{|c|c|c|c|c|c|c|c|c|c|c|}
\hline \multicolumn{11}{|c|}{$\begin{array}{c}\text { Cuestionario Lúdica y } \\
\text { Desarrollo }\end{array}$} \\
\hline Descripción & \multicolumn{2}{|c|}{ Siempre } & \multicolumn{2}{|c|}{ Generalmente } & \multicolumn{2}{|c|}{ Ocasionalmente } & \multicolumn{2}{|c|}{ Nunca } & \multicolumn{2}{|c|}{$\begin{array}{l}\text { No } \\
\text { Observado }\end{array}$} \\
\hline $\begin{array}{l}\text { 1. ¿Considera usted } \\
\text { esencial, usar } \\
\text { actividades... }\end{array}$ & 54 & $68 \%$ & 20 & $20 \%$ & 5 & $6 \%$ & 1 & $1 \%$ & 0 & $0 \%$ \\
\hline $\begin{array}{ll}2 . & \text { ¿Utiliza usted } \\
\text { actividades } \\
\text { donde el niño } \\
\text { ejercite todo su } \\
\text { cuerpo? }\end{array}$ & 46 & $58 \%$ & 24 & $24 \%$ & 9 & $11 \%$ & 1 & $1 \%$ & 0 & $0 \%$ \\
\hline
\end{tabular}




\section{Tabla 4}

Cuestionario lúdica y desarrollo (continuación)

\begin{tabular}{lccccccccc}
\hline Descripción & Siempre & Generalmente & Ocasionalmente & Nunca & $\begin{array}{c}\text { No } \\
\text { Observado }\end{array}$ \\
\hline $\begin{array}{l}\text { 3. ¿Le ofrece al niño } \\
\text { la oportunidad de } \\
\text { realizar... }\end{array}$ & 36 & $45 \%$ & 25 & $25 \%$ & 16 & $20 \%$ & 0 & $0 \%$ & $3 \%$ \\
\hline
\end{tabular}

\begin{tabular}{|c|c|c|c|c|c|c|c|c|c|c|}
\hline $\begin{array}{l}\text { 4. ¿Realiza los niños } \\
\text { actividades lúdicas } \\
\text { sin reglas? }\end{array}$ & 27 & $34 \%$ & 15 & $15 \%$ & 29 & $36 \%$ & 6 & $8 \%$ & 3 & $4 \%$ \\
\hline $\begin{array}{l}\text { 5. ¿Brinda usted a los } \\
\text { niños, la } \\
\text { oportunidad... }\end{array}$ & 50 & $63 \%$ & 25 & $25 \%$ & 2 & $3 \%$ & 2 & $3 \%$ & 1 & $1 \%$ \\
\hline
\end{tabular}

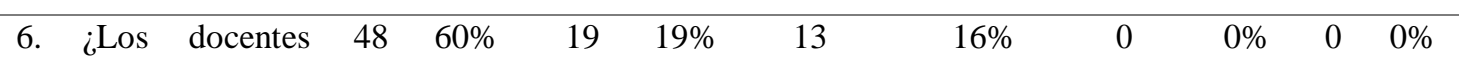
crean actividades donde el niño i...

$\begin{array}{lllllllllll}\text { 7. ¿Se crean } & 44 & 55 \% & 18 & 18 \% & 18 & 23 \% & 0 & 0 \% & 0 & 0 \%\end{array}$

lúdicas donde...

$\begin{array}{llllllllllll}\text { 8. ¿Usa } & \text { actividades } & 58 & 73 \% & 17 & 17 \% & 4 & 5 \% & 1 & 1 \% & 0 & 0 \%\end{array}$
lúdicas de expresiones plásticas (artísticas),

\begin{tabular}{|c|c|c|c|c|c|c|c|c|c|c|}
\hline $\begin{array}{l}\text { 9. ¿Usa actividades } \\
\text { como la lectura, } \\
\text { como una actividad } \\
\text { lúdica pasiva? }\end{array}$ & 31 & $39 \%$ & 20 & $20 \%$ & 7 & $9 \%$ & 10 & $13 \%$ & $2^{1}$ & $15 \%$ \\
\hline $\begin{array}{l}\text { 10. ¿Cree usted que } \\
\text { es necesario el uso de } \\
\text { actividades... }\end{array}$ & 37 & $46 \%$ & 13 & $13 \%$ & 15 & $19 \%$ & 11 & $14 \%$ & 4 & $5 \%$ \\
\hline
\end{tabular}

\begin{tabular}{|c|c|c|c|c|c|c|c|c|c|c|}
\hline $\begin{array}{l}\text { 11. ¿Cree usted que } \\
\text { las actividades } \\
\text { lúdicas... }\end{array}$ & 39 & $49 \%$ & 24 & $24 \%$ & 8 & $10 \%$ & 5 & $6 \%$ & 4 & $5 \%$ \\
\hline $\begin{array}{l}\text { 12. ¿Cree usted que } \\
\text { es importante } \\
\text { seleccionar el juego y } \\
\text { el material... }\end{array}$ & 42 & $53 \%$ & 21 & $21 \%$ & 14 & $18 \%$ & 2 & $3 \%$ & 1 & $1 \%$ \\
\hline Total, al $100 \%$ & & $51 \%$ & & $28 \%$ & & $16 \%$ & & $3 \%$ & & $3 \%$ \\
\hline
\end{tabular}

Fuente: Estudio de contexto

La población participante de la presente investigación indica que existe una correlación muy fuerte entre el uso de actividades lúdicas y el Desarrollo motor grueso, obteniéndose 
así un $51 \%$ en la categoría de siempre y un $28 \%$ en la categoría de generalmente, la cual se observa en los resultados de la "Batería Instrumental Para la medición de la Motricidad de los Niños De 4 a 5 Años; Cuestionario Lúdica y Desarrollo, así mismo se puede evidenciar que una parte de la población difiere con este resultado presentándose así un $16 \%$ en la categoría ocasionalmente y un 3\% de las misma propone que no existe una relación entre la hipótesis propuesta, por otro lado se ha evidenciado que una minúscula parte de la población encuestada un 3\% bajo la categoría de No Observado, no tiene conocimiento de cuáles son las actividades lúdicas que intervienen en el desarrollo de la motricidad gruesa.

\section{Figura 1}

Grafica de matriz de desarrollo motor grueso y actividades lúdicas

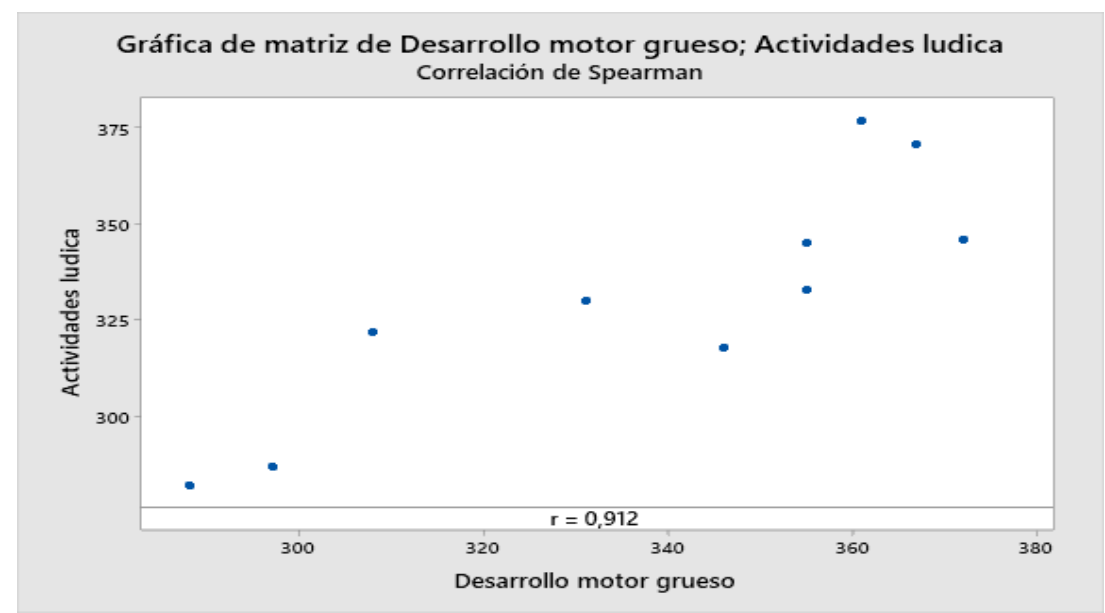

Fuente: Estudio de contexto

La presente investigación realizada a una población de 80 personas divididas entre docentes y padres fue procesada a través del programa MiniTab la cual genero la siguiente gráfica, se usó la correlación de Spearman a la cual se obtuvo un valor de 0.912 entre Las Actividades Lúdicas y el Desarrollo Motor Grueso, evidenciando que las dos variables comparten una relación directa al momento de generarse el uso de estas, dentro de un mismo proceso.

\section{Tabla 5}

Valoración de la batería instrumental para la medición de la motricidad de los niños de 4 a 5 años

Pregunta 4: Variable 1

¿camina hacia atrás?

$\begin{array}{llll}\text { Siempre } & \text { Generalmente } & \text { Ocasionalmente } & \text { Nunca }\end{array}$

Fuente: Estudio de contexto. 


\section{Figura 2}

Variable 1

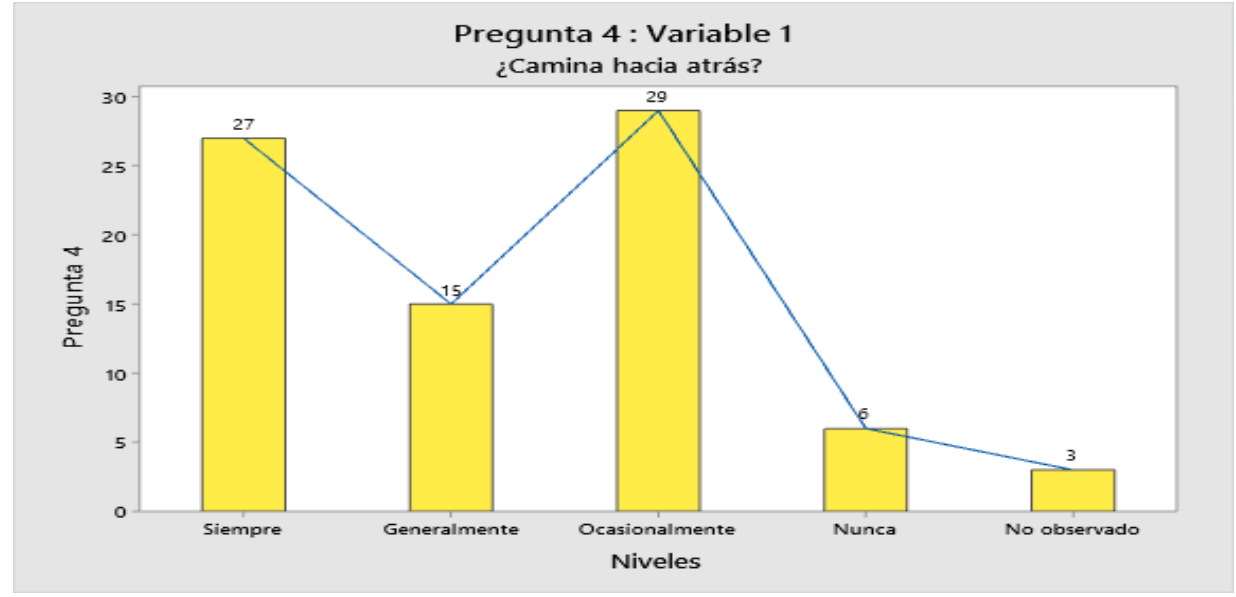

Fuente: Estudio de contexto

En la figura 2 mostramos los resultados de una de las preguntas de la batería instrumental para la medición de la motricidad de los niños de 4 a 5 años por lo tanto haremos el análisis, la pregunta fue: ¿camina hacia atrás?, a lo que los padres o tutores del niño respondieron de la siguiente manera: 29 dicen que ocasionalmente, 27 eligieron siempre, siguiendo en un orden descendente tenemos 15 generalmente, 6 dicen que nunca y finalmente 3 dicen no a verlo observado.

\section{Tabla 6}

Valoración de la batería instrumental para la medición de la motricidad de los niños de 4 a 5 años

Pregunta 12: Variable 1

¿realiza saltillos laterales hacia un lado y otro?

\begin{tabular}{ccccc}
\hline Siempre & Generalmente & Ocasionalmente & Nunca & No Observado \\
\hline 42 & 21 & 14 & 2 & 1 \\
\hline
\end{tabular}

Fuente: Estudio de contexto 


\section{Figura 3}

Variable 1

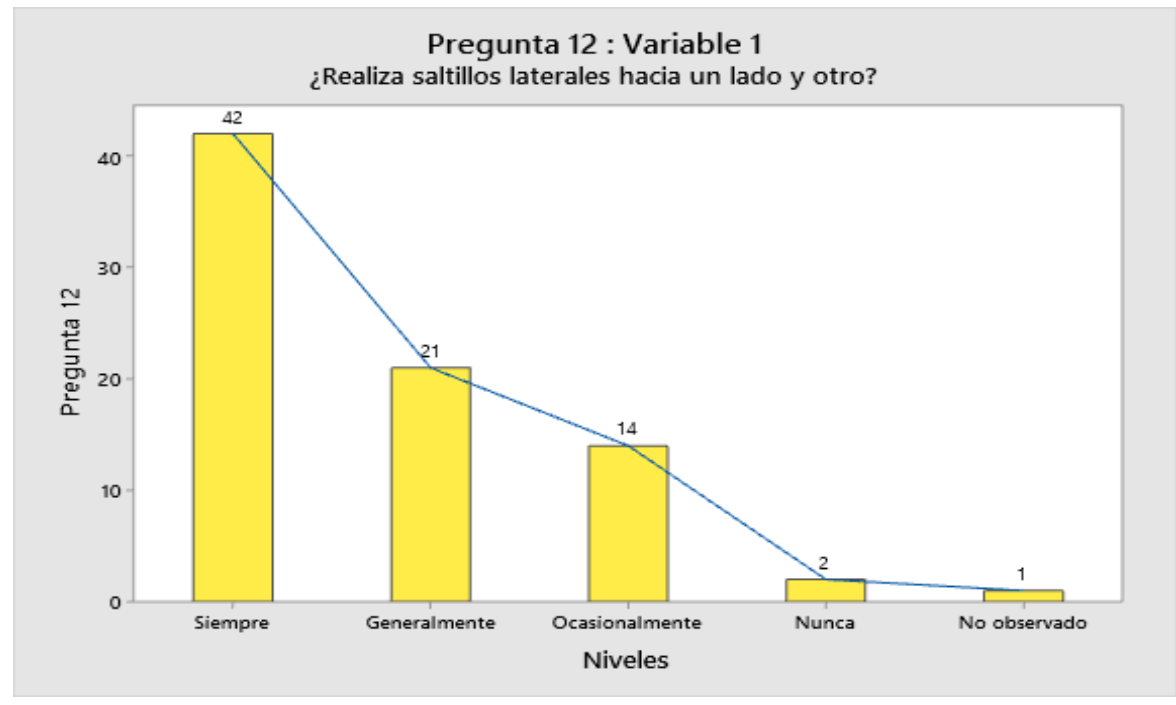

Fuente: Estudio de contexto

La figura 3 misma que pertenece a la batería instrumental para la medición de la motricidad de los niños de 4 a 5 años, arrojaron los siguientes datos a la pregunta: ¿realiza saltillos laterales hacia un lado y otro?, los encuestados según su observación 42 dice siempre, siendo este uno de los niveles más altos, por otra parte, 21 pertenece al nivel generalmente, 14 ocasionalmente, siguiendo un orden decreciente tenemos 2 elije nunca y finalmente 1 responde no observado.

Tabla 7

Cuestionario lúdica y desarrollo

Pregunta 1:

Variable 2

¿considera usted esencial, usar actividades lúdicas para el desarrollo físico del niño, en edad preescolar?

\begin{tabular}{ccccc}
\hline Siempre & Generalmente & Ocasionalmente & Nunca & No Observado \\
60 & 16 & 1 & 1 & 2 \\
\hline
\end{tabular}

Fuente: Estudio de contexto 


\section{Figura 4}

Variable 2

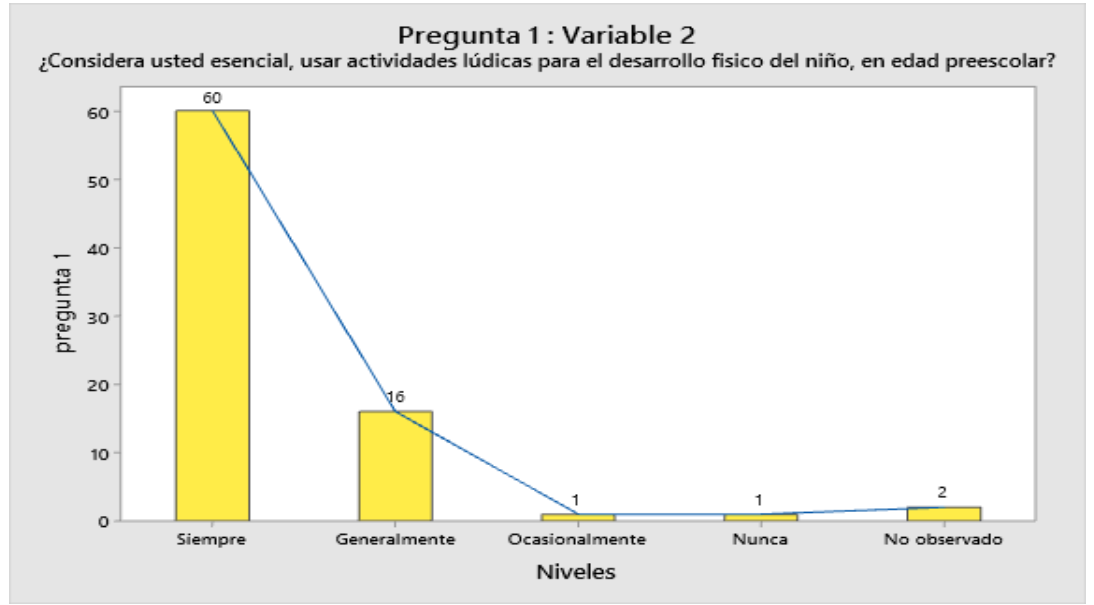

Fuente: Estudio de contexto

En la figura 4 tenemos datos del cuestionario de contribución de las actividades lúdicas y la pregunta uno decía lo siguiente: ¿considera usted esencial, usar actividades lúdicas para el desarrollo físico del niño, en edad preescolar?, a lo que los encuestados responden de la siguiente manera: 60 dice que siempre va hacer esencial utilizar dichas actividades lúdicas este es uno de los porcentajes más altos en comparación con los demás, 16 menciona generalmente, 2 eligen el nivel no observado y para finalizar tenemos una igualación de 1 tanto para ocasionalmente como para nunca respectivamente.

\section{Tabla 8}

Cuestionario lúdica y desarrollo

\section{Pregunta 10:}

Variable 2

¿Cree usted que es necesario el uso de actividades lúdicas cooperativas, para mejorar la relación entre los niños?

\begin{tabular}{ccccc} 
Siempre & Generalmente & Ocasionalmente & Nunca & $\begin{array}{c}\text { No } \\
\text { Observado }\end{array}$ \\
30 & 29 & 17 & 1 & 3 \\
\hline
\end{tabular}

Fuente: Estudio de contexto 


\section{Figura 5}

Variable 2

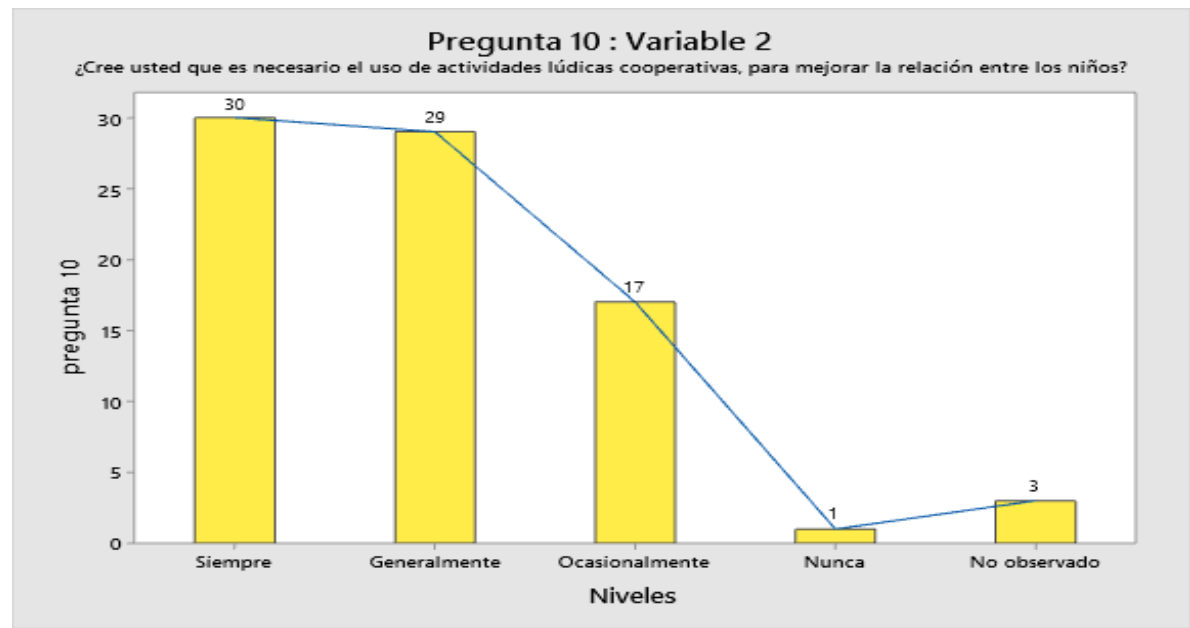

Fuente: Estudio de contexto

La figura 5 nos muestra resultados del cuestionario de Contribución de las actividades lúdicas y la pregunta numero 12 menciona lo siguiente: ¿Cree usted que es necesario el uso de actividades lúdicas cooperativas, para mejorar la relación entre los niños?, los padres o tutores del niño o niña responden de esta manera: 30 establece que siempre, 29 dice que generalmente, por otra parte, 17 escoge la opción ocasionalmente, 3 establece la no observación y finalmente 1 pertenece a nunca.

\section{Conclusiones}

- Se concluye que es necesario realizar un diagnóstico inicial en donde se valoren los procesos de desarrollo motriz grueso, esto permitirá atender las necesidades particulares y brindar un seguimiento individualizado que responda a las características evolutivas de los infantes.

- Si existe una estrecha relación entre la aplicación de la lúdica y el desarrollo motor grueso de niños entre 4 y 5 años ya que al exigir movimientos corporales mejora el equilibrio, coordinación, tonificación muscular, y en general los movimientos gruesos del cuerpo.

- Es necesario desarrollar en los niños de 4 y 5 años la coordinación dinámica general, a través del movimiento lúdico, ya que el niño disfruta del juego y es a partir de estas actividades que se fortalece el tono muscular y control postural.

- La lúdica favorece el desarrollo cognitivo y social de los seres humanos desde edades muy tempranas, fortalece la capacidad intelectual ayuda a potenciar los valores humanos y otras habilidades como la motricidad, inteligencia, creatividad y sociabilidad. 


\section{Referencias bibliográficas}

Balseca, C., \& Grace, M. (2016). El juego y la motricidad gruesa en niños de 3 a 4 años del centro de educación inicial Lucia Franco de Castro de la parroquia de Conocoto. Quito.

Calero, S., Garzón, B., \& Chávez, E. (2020). La correlación compensación en niños sordociegos con alteraciones motrices a través de actividades físicas adaptadas. Scielo.

Chocce, E., \& Conde, D. (2018). Juegos Populares para desarrollar la motricidad gruesa en niños de 5 años. Huancavelica.

Dente, L., Gonzales, A., Weinstein, E., \& Brounstein, S. (2018). La Centralidad del juego en la Educación Inicial. Buenos Aires.

Diaz, R., Rosales, L., \& Quincoces, R. (2014). Guía metodológica de juegos para el desarrollo de habilidades motrices básicas en escolares.

Gutiérrez, M. (2013). Actividades sensorio-motrices para la lectoescritura. Barcelona: Inde.

Rojas Estela y Sara Judith. (2018). El juego como estrategia para mejorar la motricidad gruesa en los niños de 5 años. Chiclayo.

Lupuche, K. (2017). Las actividades lúdicas y el desarrollo de la motricidad gruesa en niños de 4 años. Lima.

Macias, E. (2017). La interacción comunicativa y la convivencia escolar en el aula de la educación secundaria. Extremadura.

Preciado. (2019). Juegos lúdicos basados en el enfoque significativo mejora el desarrollo de la motricidad en niños de 4 años. Tumbes.

Ramírez, D. (2019). Los juegos lúdicos como recurso para desarrollar la motricidad gruesa en niños de cinco años. Huallanga.

Rigal, R. (2016). Educación motriz y Educación Psicomotriz. España: Producciones S.A. Rigal, R. (2016). Educación motriz y educación psicomotriz en Preescolar y Primaria. España: INDE Publicaciones.

Sanjinez, A. (2018). El juego como estrategia pedagógica. Tumbes.

Vera, M. (2019). Estrategias lúdicas en el desarrollo de la motricidad gruesa en los niños de 3 a 4 años. 
Camargo, E., \& Maciel, R. (2016). La importancia de la Psicomotricidad en la Educación de los niños. Brasilia: Base de conocimiento.

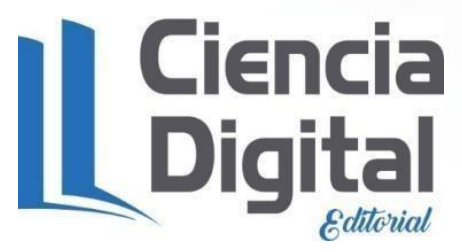

El artículo que se publica es de exclusiva responsabilidad de los autores y no necesariamente reflejan el pensamiento de la Revista Conciencia Digital.

\section{Ciencia}

El artículo queda en propiedad de la revista y, por tanto, su publicación parcial y/o total en otro medio tiene que ser autorizado por el director de la Revista Conciencia Digital.
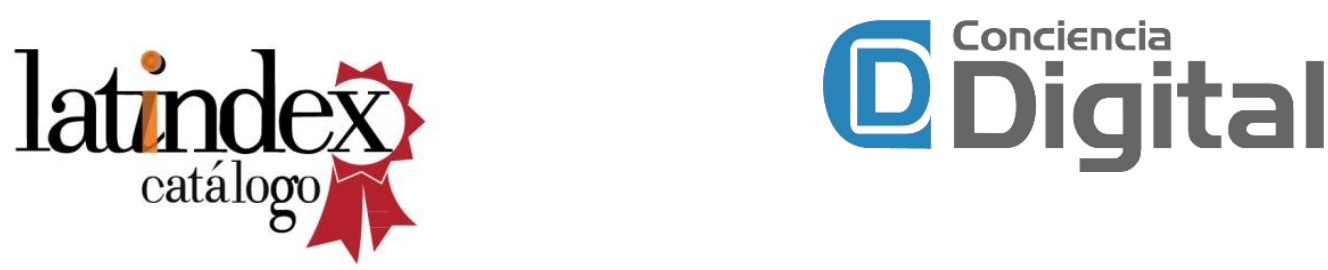

Indexaciones

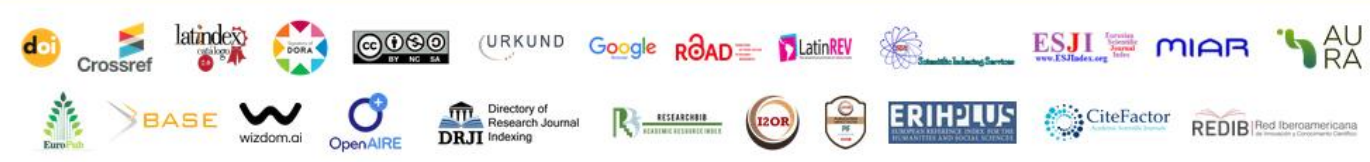

